\title{
Emociones del profesorado de educación física: revisión narrativa (2010-2020) Physical education teacher emotions: narrative review (2010-2020)
}

\author{
*Felipe Nicolás Mujica Johnson, **Nelly Del Carmen Orellana Arduiz \\ *Universidad Católica de Temuco (Chile), **Universidad de Playa Ancha (Chile)
}

\begin{abstract}
Resumen. El estudio de las emociones en educación física es cada vez más común. Sin embargo, las emociones del alumnado han gozado de más protagonismo en la literatura, en comparación con las del profesorado. El estudio de las emociones del profesorado se divide en dos enfoques principales, uno orientado a la intensidad y otro a la calidad emocional. Con la finalidad de contribuir a la comprensión de la calidad emocional, se ha decidido realizar una revisión narrativa que tuvo por objetivo analizar los significados de las emociones del profesorado de educación física. La búsqueda se llevó a cabo en siete bases de datos internacionales y se lograron identificar siete investigaciones cualitativas sobre la temática. Los resultados indican que las emociones del profesorado responden al saber docente, los valores, el desempeño laboral, la experiencia personal y profesional. Se concluye que las emociones del profesorado expresan la complejidad de la labor docente y sus posicionamientos éticos frente a diversas situaciones pedagógicas.
\end{abstract}

Palabras clave: emoción, ética, profesorado, educación física, investigación cualitativa.

\begin{abstract}
The study of emotions in physical education is increasingly common. However, the emotions of the students have enjoyed more prominence in the literature, compared to those of the teachers. The study of teachers' emotions is divided into two main approaches, one focused on intensity and the other on emotional quality. With the intention of contributing to the understanding of emotional quality, it has been decided to carry out a narrative review that aimed to analyze the meanings of the emotions of physical education teachers. The search was carried out in seven international databases and it was possible to identify seven qualitative investigations on the subject. The results indicate that teachers' emotions respond to teaching knowledge, values, work performance, personal and professional experience. It is concluded that the teachers' emotions express the complexity of the teaching work and their ethical positions in the face of diverse pedagogical situations.
\end{abstract}

Key words: emotion, ethics, teachers, physical education, qualitative research.

\section{Introducción}

En las últimas décadas se está apreciando un cambio sustancial en el ámbito educativo, el cual hace referencia a la reivindicación de la afectividad en el proceso de enseñanzaaprendizaje (Bisquerra, 2003, 2005; Bisquerra \& Filella, 2018; Marina, 2005, 2016; Mujica, 2018; Sonlleva, Martínez \& Monjas, 2018). Algunos autores han denominado este cambio como el giro afectivo de la educación (Escolano, 2018; Zembylas, 2019). No obstante, el tema de la afectividad y, en concreto, de las emociones, no es para nada nuevo en el pensamiento occidental, ya que viene siendo discutido desde los inicios de la filosofía y se ha mantenido presente a lo largo de su historia (Casado \& Colomo, 2006). En este recorrido ha habido diferentes posicionamientos teóricos frente al tema, destacando la corriente fenomenológica, que defendió su importancia y autonomía en la vida efectiva de las personas (Mujica, 2020; Scheler, 2005; Stein, 2007; Vendrell, 2015; von Hildebrand, 2009).

Sabiendo que la discusión en torno a las emociones humanas tiene un largo recorrido, hemos de mencionar que el giro afectivo no representa un descubrimiento de la importancia de la afectividad, sino que, como bien explica Zaccagnini (2008), significa, en el caso de la emoción, la aceptación de un concepto de emoción que se desprende de la interpretación racionalista. Es decir, del significado que las considera como pasiones que perturban el intelecto. Por cierto, este giro afectivo también se ha visto beneficiado con las teorías constructivistas y postpositivistas que han señalado el rol del sentir en el aprendizaje (Ausubel, 2002;

Fecha recepción: 29-05-19. Fecha de aceptación: 20-07-20

Felipe Nicolás Mujica Johnson

fmujica@live.cl
Sarramona, 2008; Vygostsky, 1995). Lo anterior también se ha visto reflejado en el ámbito de la educación física $(\mathrm{EF})$, sobre todo, en el anhelo de una educación integral (Cagigal, 1996; Devís, 2018; Kirk, 2010; Mujica \& Orellana, 2019; Velázquez \& Hernández, 2010). Sobre el término emoción, Zaccagnini (2008) bien plantea el siguiente:

Una compleja combinación de procesos corporales, perceptivos y aptitudinales que producen en las personas un estado psicológico global, que puede ser positivo o negativo, de poca o mucha intensidad y de corta o larga duración, o mezclas de esos elementos, y que genera una expresión gestual que puede ser identificada por las demás personas (p. 48).

En el contexto del giro afectivo en la $\mathrm{EF}$, en los últimos años se han realizado diferentes investigaciones empíricas sobre las emociones en dicha asignatura, como lo muestran dos estudios de revisión centrados, principalmente, en el sentir del alumnado (Mujica, Orellana \& Concha, 2017; Bermúdez \& Saenz-López, 2019). En los estudios sobre las emociones en EF podemos reconocer que existen dos tipos de aproximación, uno de carácter cuantitativo referido a la intensidad emocional (Pic, Lavega-Burgués, MuñozArroyave, March-Llanes \& Echeverri-Ramos, 2019; Zamorano-García, Gil-Madrona, Prieto-Ayuso \& ZamoranoGarcía, 2018) y otro de carácter cualitativo referido a la calidad emocional (Alves, MacPhail, Queirós \& Batista, 2019; Leisterer \& Jekauc, 2019; Monforte \& Pérez-Samaniego, 2017; Salgado-López, 2014). Evidentemente, ambos métodos pueden contribuir de diferente forma a la mejora educativa en dicha asignatura pedagógica. En estas corrientes científicas ha existido una dimensión transversal, que es la del bienestar subjetivo (BS) (Diener, Lucas \& Oishi, 2018; Myers \& Diener, 1995, 2018). En dicho bienestar las emociones tendrían un rol importante, siendo valoradas en positivas y ne- 
gativas. Aquella valoración hace referencia a que «una emoción positiva es el resultado de una valoración favorable del $\mathrm{BS}$, mientras que una emoción negativa es el resultado de una valoración desfavorable» (Mujica-Johnson \& Jiménez, 2019,p. 161).

Los estudios sobre las emociones del profesorado de EF han tenido menor atención en algunos estudios de revisión (Mujica, Orellana \& Concha, 2017; Bermúdez \& Saenz-López, 2019), de modo que para este trabajo se ha decidido colocar el foco en dicho grupo social y profesional. Específicamente, se ha decidido realizar una aproximación a las investigaciones referidas a la calidad emocional porque, siguiendo algunos postulados de la corriente fenomenológica (Fernández, 2013; Vendrell, 2015; von Hildebrand, 2009), se considera que es fundamental comprender los objetos intencionales que suscitarían las emociones. Dichos objetos podrían tener un sentido cultural, psicológico, ético o biológico. En este sentido, el presente estudio de revisión tiene por objetivo analizar los significados de las emociones del profesorado de EF que han sido interpretados en diferentes estudios cualitativos.

\section{Material y método}

Con la intención de profundizar en la calidad emocional, se ha optado por una aproximación de carácter cualitativo al fenómeno emocional del profesorado de EF y, a su vez, a un estudio de revisión narrativa (Franco, Machado, Grinbaum \& Porfirio, 2019; Mujica et al., 2017). Este tipo de revisión favorece el análisis de los significados emocionales. Además, a diferencia de la revisión sistemática, tiene una estructura más abierta y flexible, la cual no exige seguir un proceso estructurado y lineal (Rother, 2007). Por ello, esta revisión no expone en forma exhaustiva el proceso de búsqueda bibliográfica y los antecedentes de cada investigación. No obstante, sí se presentan los principales pasos seguidos para dicha búsqueda y algunos antecedentes relevantes de los estudios seleccionados.

Esta revisión contempla la búsqueda de investigaciones en siete bases de datos, específicamente, en Web of Science (WOS), Scopus, Scientific Electronic Library Online (SciELO), Red de Científicas de América Latina y el Caribe, España y Portugal (REDALYC), Dialnet, Google Académico y Directory of Open Access Journals (DOAJ). Los términos para la búsqueda bibliográfica fueron profesorado de Educación Física y emociones en castellano e inglés. En el caso de la base de datos WOS, la consulta se realizó como búsqueda avanzada, utilizando la fórmula para títulos. En cuanto a los criterios de búsqueda, se tuvieron los siguientes: a) investigaciones empíricas con metodología cualitativa; b) investigaciones publicadas en revistas científicas entre los años 2010 y 2020; c) investigaciones de todos los idiomas; y d) investigaciones sobre las emociones de docentes en servicio en el contexto escolar de cualquier contexto geográfico. Para este estudio se incluyeron solo estudios con docentes titulados, no estudiantes de pedagogía en EF.

La búsqueda bibliográfica permitió identificar y seleccionar siete investigaciones que respondían a los criterios establecidos, de las cuales se extrajo la siguiente información: a) autores; b) año de publicación; c) país; d) cantidad de parti- cipantes y su sexo; y e) principales resultados. Posteriormente, los principales resultados fueron sometidos a un análisis de contenido inductivo (Osses, Sánchez \& Ibáñez, 2006), el cual permitió categorizar los hallazgos y asociarlos en el software de análisis de datos cualitativos Atlas.ti 7.5.

\section{Resultados y discusión}

En términos generales, los siete estudios identificados se han desarrollado en cuatro naciones de tres diferentes continentes. Es sorprendente la poca cantidad de investigaciones cualitativas sobre la temática indagada, sobre todo, porque se consideró un rango de año bastante amplio. Para exponer los primeros antecedentes de las investigaciones seleccionadas se ha elaborado la Tabla 1, la cual presenta una descripción general de los estudios incluidos en esta revisión.

Con respecto a los principales hallazgos de las emociones del profesorado de EF en los estudios seleccionados, se pueden apreciar en la Tabla 2.

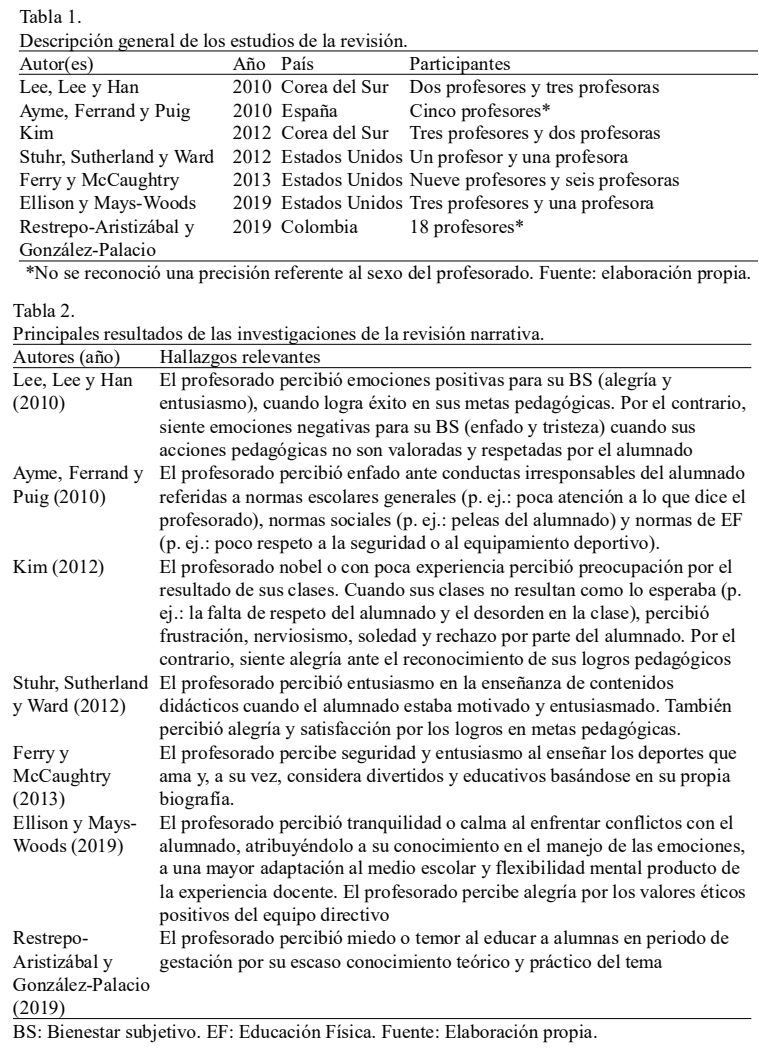

Los hallazgos descritos anteriormente, producto del análisis de contenido, dieron lugar a las siguientes cuatro categorías de motivos que suscitaron emociones en el profesorado de EF: a) saber y experiencia docente; b) desempeño docente; c) valores éticos; y d) refuerzo positivo.

\section{Saber y experiencia docente}

Esta categoría hace referencia a la influencia del conocimiento de temas específicos transversales a la educación y de la experiencia personal en las emociones del profesorado de EF. Sobre el conocimiento aludido, hemos de destacar que sería de carácter teórico y práctico (Rauner, 2007). En el estudio de Ellison \& Mays-Woods (2019), el profesorado señaló sentir emociones positivas para su BS por su conoci- 
miento sobre competencias emocionales. En sentido contrario, el estudio de Restrepo-Aristizábal \& González-Palacio (2019) señaló que el profesorado sintió emociones negativas para su BS por la falta de conocimientos sobre las alumnas en etapa de gestación en EF.

Cabe destacar, que el conocimiento práctico se consigue con la experiencia profesional (Luis-Pascual, 2008), de modo que eso podría explicar las emociones de preocupación y nerviosismo del profesorado nobel (Kim, 2012); de temor por la inexperiencia en temas concretos presenten en la sesión de EF (Restrepo-Aristizábal \& González-Palacio, 2019); y de seguridad en la enseñanza de contenidos didácticos con que los el profesorado se encuentra familiarizado (Ellison \& Mays-Woods, 2019; Ferry \& McCaughtry, 2013). Estos hallazgos se asemejan a los resultados de otras investigaciones en EF que han mostrado una relación de la cultura deportiva como de la experiencia motriz en las emociones del alumnado escolar y universitario (Duran et al., 2015; Monforte \& Pérez-Samaniego, 2017; Mujica-Johnson \& Jiménez, 2019, 2020; Pic et al., 2019).

\section{Desempeño docente}

Esta categoría representa la influencia que, desde la conciencia emocional del profesorado, tuvo el éxito o fracaso de las metas pedagógicas en sus emociones. En concreto, el profesorado señaló sentir emociones positivas para su BS en los logros deseados y negativas para su BS en los resultados no deseados o en la ausencia de logros. Por ejemplo, algunos logros son lograr captar la atención del alumnado y lograr implicar al alumnado en los aprendizajes planteados (Ayme et al., 2010; Kim, 2012; Lee et al., 2010; Stuhr et al., 2012).

La relación de los éxitos y fracasos en las metas de logro también ha sido reconocida en diferentes estudios sobre emociones del alumnado escolar en EF (Duran et al., 2015; Miralles, Filella \& Lavega, 2017; Pic et al., 2019). Además, dichas emociones parecen ser el reflejo secundario de una intencionalidad ética para con el acto pedagógico y la contribución en el desarrollo personal del alumnado (Fernández, 2013; Mujica \& Orellana, 2021).

\section{Valores éticos}

La presente categoría es representativa para la incidencia que habrían tenido los valores éticos del propio profesorado y de los demás integrantes del proceso educativo en sus emociones. Las emociones positivas para el BS se asociaron a la presencia de solidaridad, honestidad, responsabilidad y respeto en los centros educativos (Lee et al., 2010; Kim, 2012; Ellison \& Mays-Woods, 2019). En sentido contrario, el profesorado habría sentido emociones negativas para el BS ante la presencia de valores éticos negativos en el proceso de enseñanza-aprendizaje en EF (Ayme et al., 2010; Lee et al., 2010). El temor del profesorado por su falta de conocimiento e inexperiencia en la educación con alumnas en periodo de gestación también podría ser interpretado desde la perspectiva ética, ya que podría explicar la intención de hacer daño a otra persona (Restrepo-Aristizábal \& GonzálezPalacio, 2019).

Estos resultados serían consecuentes con los argumentos filosóficos que, en ocasiones, señalan a las emociones como respuestas a valores éticos positivos y negativos (Fernández, 2013; Scheler, 2005; Vendrell, 2015; von Hildebrand, 2009). Otros estudios en el marco universitario de EF también han reflejado la relación de los valores éticos presentes en la cultura escolar y las emociones del estudiantado (Alves et al., 2019; Mujica-Johnson \& Jiménez, 2019, 2020).

\section{Refuerzo positivo}

La cuarta y última categoría, representa los actos que el profesorado interpretó como de reconocimiento a su buena labor docente, los cuales serían expresados de forma directa o indirecta. Así, dichos actos suscitarían emociones positivas para su BS. Entre los actos de refuerzo positivo directo, se encuentran las felicitaciones explícitas por el buen desempeño docente (Kim, 2012). Por otro lado, entre los actos de refuerzo positivo indirecto, se encontraría la expresión de emociones positivas para el BS del propio alumnado como consecuencia de las acciones pedagógicas del profesorado (Stuhr et al., 2012).

La relación del refuerzo positivo con las emociones positivas para el BS coincidiría con diferentes estudios en el ámbito de la EF, incluyendo los estudios sobre la teoría de las metas de logro que lo asocian a un clima orientado a la tarea (Cabello, Moyano \& Tabernero, 2018; Escamilla, Alguacil \& Huertas, 2020; Timken, McNamee \& Sarah, 2019). En este sentido, las relaciones humanas de amistad y fraternidad podrían ser muy importantes para la afectividad positiva del profesorado (Méndez-Giménez, García-Romero \& Cecchini-Estrada, 2018; Mujica-Johnson \& Jiménez, 2020; Salgado-López, 2014), lo cual, evidentemente, requiere ser investigado con mayor profundidad y especificidad.

A modo de síntesis, en la Figura 1 se expone un esquema conceptual de las categorías y su relación con las emociones en función del BS.

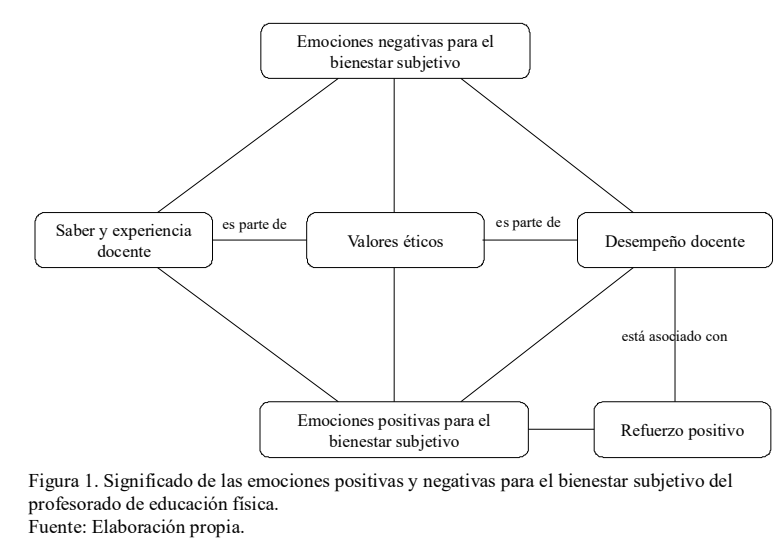

\section{Conclusiones y aplicaciones prácticas}

Esta revisión muestra la complejidad que podría significar para el profesorado gestionar una sesión de EF, sobre todo, cuando existen escenarios pedagógicos que presentan diversas problemáticas éticas y socioculturales. En dicho contexto, las emociones positivas y negativas para el BS del profesorado, en diferentes ocasiones, representarían su posicionamiento ético-moral y su disposición para promo- 
ver mejoras educativas.

Con respecto a las atribuciones emocionales, se concluye que las emociones del profesorado de EF han sido suscitadas por la falta de conocimiento y experiencia profesional, por la mala conducta del alumnado, por contagio emocional, por la auto-percepción del desempeño docente, al igual que por el refuerzo positivo directo e indirecto de parte de la comunidad educativa. También fueron relevantes los valores éticos implícitos en la cultura escolar.

En cuanto a las aplicaciones prácticas, se reconocen las siguientes tres: a) el profesorado universitario formador de docentes en EF puede explicar estos significados emocionales a su alumnado y reflexionar con él sobre los aspectos que pueden ser mejorados en la formación inicial docente; b) el profesorado de EF en servicio activo en centros de educación escolar, puede analizar estos significados y reflexionar sobre la intencionalidad de sus propias emociones en el aula y en los otros espacios del entorno escolar; y c) el mismo profesorado de EF escolar puede socializar los significados emocionales con su alumnado y suscitar su reflexión sobre la conciencia que tienen de la afectividad de sus docentes.

\section{Futuras líneas de investigación}

Consideramos que el poco interés por las emociones del profesorado de EF podría ser perjudicial para la mejora de las prácticas pedagógicas en dicha asignatura, ya que la educación integral debe incluir a toda la comunidad educativa, no solo al alumnado. Además, estas investigaciones requieren ser aumentadas a nivel global para contribuir en la comprensión de la afectividad del profesorado porque aportan la base científica necesaria para la formación docente del magisterio de esta disciplina pedagógica.

Para responder a la escasa investigación sobre el tema, se propone el desarrollo de nuevas investigaciones cualitativas, con la finalidad de aportar conocimiento sobre la calidad emocional. Podría ser muy útil realizar estudios que asocien las emociones del profesorado con las siguientes dimensiones: a) experiencia docente; b) conocimiento de diferentes materias asociadas a la asignatura; c) sexo; d) género; e) cultura escolar; f) liderazgo ético; g) justicia social; h) contenidos didácticos; i) biografía personal del profesorado; j) convivencia general del centro educativo; k) especialización curricular; 1) bienestar subjetivo; y m) entorno sociocultural del centro educativo. Cabe mencionar, que cada una de estas dimensiones podría ser investigada de forma independiente, ya sea en estudio de casos individuales o colectivos. Por otro lado, algunas de las dimensiones mencionadas podrían ser indagadas de forma conjunta. Otra opción es contrastar los resultados de diferentes grupos de profesorado en función de una o más dimensiones. Por ejemplo, se podría comparar los resultados del profesorado hombre y mujer; con diferentes especializaciones curriculares; o perteneciente a centros educativos con diferentes entornos socioculturales.

\section{Referencias}

Alves, M., MacPhail, A., Queirós, P., \& Batista, P. (2019). Becoming a physical Education Teacher during formalized school placement:
A rollercoaster of emotions. European Physical Education Review, 25(3), 893-909. doi: 10.1177/1356336X18785333

Ausubel, D. (2002). Adquisición y retención del conocimiento: una perspectiva cognitiva. Barcelona: Paidós.

Ayme, S., Ferrand, C., \& Puig, N. (2010). Provocaciones del alumnado y emoción de ira en el profesorado de Educación Física. El caso de los centros de atención educativa preferente de la ciudad de Barcelona. Apunts. Educación Física y Deportes, 102, 22-30.

Bermúdez, C., \& Sáenz-López, P. (2019). Emociones en Educación Física. Una revisión bibliográfica (2015-2017). Retos. Nuevas Tendencias en Educación Física, Deportes y Recreación, 36, 597-603.

Bisquerra, R. (2003). Educación emocional y competencias básicas para la vida. Revista de Investigación Educativa, 21(1), 7-43.

Bisquerra, R. (2005). La educación emocional en la formación del profesorado. Revista Interuniversitaria de Formación del Profesorado, 19(3), 95-114.

Bisquerra, R., \& Filella, G. (2018). Análisis del vocabulario emocional en el profesorado de lengua. Revista Electrónica Interuniversitaria de Formación del Profesorado, 21(1), 161-172. doi: 10.6018/ reifop.21.1.298421

Cabello, A., Moyano, M. y Tabernero, C. (2018). Procesos psicosociales en Educación Física: actitudes, estrategias y clima motivacional percibido. Retos. Nuevas Tendencias en Educación Física, Deportes y Recreación, 34, 19-24. Recuperado de: https://fecyt.fecyt.es / index.php/retos/article/view/57668

Cagigal, J. M. (1996). Obras Selectas. Volumen III. Madrid: Comité Olímpico Español.

Casado, C. y Colomo, R. (2006). Un breve recorrido por la concepción de las emociones en la Filosofia Occidental. A Parte Rei, 47, 1-10.

Castejón, F. J., Giménez, F. J., Jiménez, F., \& López, V. (2003). Iniciación deportiva. La enseñanza y el aprendizaje comprensivo en el deporte. Sevilla: Wanceulen.

Devís, J. (2018). Los discursos sobre las funciones de la Educación Física Escolar. Continuidades, discontinuidades y retos. Revista Española de Educación Física y Deportes, 423, 121-131.

Diener, E., Lucas, R., \& Oishi, S. (2018). Advances and open questions in the science of subjective well-being. Collabra: Psychology, 4(1), $1-49$.

Duran, C., Lavega, P., Salas, C., Cristofol, T., Tamarit, M., \& Invernó, J. (2015). Educación física emocional en adolescentes. Identificación de variables predictivas de la vivencia. Cultura, Cienciay Deporte, 10(28), 5-18. doi: 10.12800/ccd.v10i28.511

Ellison, D., \& Mays-Woods, A. (2019). In the face of adversity: four physical educator's experiences of resilience in high-poverty schools. Physical Education and Sport Pedagogy, 24(1), 59-72. doi:17408989.2018.1536201

Escamilla, P., Alguacil, M. y Huertas, M. (2020). Variables predictoras de la motivación deportiva en estudiantes de secundaria federados y no federados. Retos. Nuevas Tendencias en Educación Física, Deportes y Recreación, 38, 58-65.

Escolano,A. (2018). The affective turn in the history of human formation. A memory of schooling and emotions. Historia y Memoria de la Educación, 7,391-422. doi: 10.5944/hme.7.2018.19101

Fernández,P.(2013). Sobre la intencionalidad secundaria de las emociones. Diánoia, 58(70), 3-34. doi: 10.21898/dia.v58i70.117

Ferry, M., \& McCaughtry, N. (2013). Secondary Physical Educators and Sport Content: A Love Affair. Journal of Teaching in Physical Education, 32, 375-393. doi: 10.1123/jtpe.32.4.375

Franco, R., Machado, J., Grinbaum, R., \& Porfirio, G. (2019). Barriers to outpatient education for medical students: a narrative review. International Journal of Medical Education, 10, 180-190. doi:10.5116/ijme.5d76.32c5

Kirk, D. (2010). Physical Education Futures. New York: Routledge.

Kim, N. (2012). Exploring the emotions of new physical education teachers at secondary school. Journal of Korean Sports Education Society, 19(3), 69-84. 
Lee, J., Lee, S., \& Han, K. (2010). A Study on the Elementary School Teachers's Emotion in the light of Physical Education Class. The Korean Journal of Sports Science, 19(1), 603-619.

Leisterer, S., \& Jekauc, D. (2019). Students' emotional experience in Physical Education. A qualitative study for new theoretical insights. Sports, 7, 1-15. doi: 10.3390/sports7010010

Luis-Pascual, J. C. (2008). Diagnóstico y perfil formativo del docente de Educación Física escolar. Madrid: Adal.

Marina, J. (2005). Precisiones sobre la educación emocional. Revista Interuniversitaria del Profesorado, 54, 27-44.

Marina, J. (2016). Vence tus miedos: cuaderno de actividades. Barcelona:Ariel.

Méndez-Giménez,A., Fernández-Río, J., \& Méndez-Alonso, D. (2015). Sport Education Model Versus Traditional Model: Motivational and Sportsmanship Effects. Revista Internacional de Medicina y Ciencias de la Actividad Físicay del Deporte, 15(59), 449-466. doi: 10.15366/rimcafd2015.59.004

Méndez-Giménez, A., García-Romero, C., \& Cecchini-Estrada, J. A. (2018). 3x2 Achievement Goals, Friendship and Affectivity in Physical Education: Age-Gender Differences. Revista Internacional de Medicina y Ciencias de la Actividad Física y el Deporte, 18 (72), 637-653. doi: 10.15366/rimcafd2018.72.003

Miralles, R., Filella, G., \& Lavega, P. (2017). Educación Física emocional a través del juego en educaciónprimaria. Ayudando a los maestros a tomar decisiones. Retos. Nuevas Tendencias en Educación Física, Deportey Recreación, 31, 88-93.

Monforte, J., \& Pérez-Samaniego, V. (2017). El miedo en Educación Física: Una historia reconocible. Movimento, 23(1), 85-99. doi: $10.22456 / 1982-8918.71272$

Mujica, F. (2018). Educar y suscitar emociones en la educación: Análisis crítico de su contribución al desarrollo moral. Ensayos. Revista de la Facultad de Educación de Albacete, 33(2), 15-27. doi: 10.18239/ensayos.v33i2.1540.

Mujica, F. (2020). El término Educación Física en la posmodernidad: contribución de algunas perspectivas fenomenológicas. Retos. Nuevas Tendencias en Educación Física, Deportes y Recreación, 38, 795-801. Recuperado de: https://recyt.fecyt.es/index.php/retos/ article/view/73011/49514

Mujica, F., \& Orellana, N. (2019). Emociones en educación física desde la perspectiva constructivista: análisis de los currículos de España y Chile. Praxis \& Saber, 10(24), 297-319. doi: 10 . 19053 / 22160159.v10.n25.2019.8468

Mujica, F., \& Orellana, N. (2021). El amor en educación física: una perspectiva filosófica basada en Max Scheler y José Ortega y Gasset. Retos. Nuevas Tendencias en Educación Física, Deportes y Recreación, 39, 1-6. Recuperado de: https://recyt.fecyt.es/ index.php/retos/article/view/78787

Mujica, F., Orellana, N., \& Concha, R. (2017). Emociones en la clase de Educación Física: revisión narrativa (2010-2016). Ágora para la Educación Física y el Deporte, 19(1), 119-134. doi: 10.24197/aefd.1.2017.119-134

Mujica-Johnson, F., \& Jiménez, A. C. (2019). Percepción emocional en la asignatura de Baloncesto de estudiantes del Grado en Ciencias del Deporte: Estudio piloto. Cuadernos de Psicología del Deporte, 19(2), 152-166. doi: 10.6018/cpd.347161

Mujica-Johnson, F., \& Jiménez, A. C. (2020). Percepción emocional del alumnado de $3^{\circ}$ de ESO ante las prácticas de la unidad didáctica de baloncesto en Educación Física. Revista Española de Educación Física y Deportes, 429, 47-60.

Myers, D., \& Diener, E. (1995). Who is Happy? Psychological Science, 6(1), 10-19. doi: 10.1111/j.1467-9280.1995.tb00298.x

Myers, D., \& Diener, E. (2018). The scientific pursuit of happiness. Perspectives on Psychological Science, 13(2), 218-225. doi: $10.1177 / 1745691618765171$

Osses, S., Sánchez, I., \& Ibáñez, F. (2006). Investigación cualitativa en educación. Hacia la generación de teoría a través del proceso analítico. Estudios Pedagógicos, 32(1), 119-133. doi: 10.4067/ s0718-07052006000100007

Pic, M., Lavega-Burgués, P., Muñoz-Arroyave, V., March-Llanes, J., \& Echeverri-Ramos, J.A. (2019). Predictive variables of emotional intensity and motivational orientation in the sports initiation of basketball. Cuadernos de Psicología Del Deporte, 19(1), 241-251. doi: $10.6018 / \mathrm{cpd} .343901$

Rauner, F. (2007). El conocimiento práctico y la competencia de acción en la profesión. Revista Europea de Formación Profesional, 40, 58-73.

Restrepo-Aristizábal, L.F., \& González-Palacio, E. V. (2019). Relación pedagógica entre el profesor y la joven gestante en la clase de Educación Física. Prospectiva, 27, 241-266. doi: 10.25100/ prts.v0i27.7278

Rother,E. (2007). Revisión sistemática x revisión narrativa. Acta Paulista de Enfermagem, 20(2), 9-10. Recuperadode:https://ww.scielo.br/ pdf/ape/v20n2/es_a01v20n2.pdf

Salgado-López, J. I. (2014). As emoções como condicionante didático no ensino dos deportes sociomotores de colaboração-oposição: estudo dos problemas afetivos ligados ao contacto en rugby, andebol e voleibol.(Tesis Doctoral no publicada). Universidade Da Coruña, LaCoruña.

Sarramona, J. (2008). Teoría de la educación. Barcelona:Ariel.

Scheler,M.(2005). Esenciay formas de la simpatía. Sígueme: Salamanca.

Sonlleva, M., Martínez, A., \& Monjas, R. (2018). Los procesos de evaluación y sus consecuencias. Análisis de las experiencias del profesorado de Educación Física. Estudios Pedagógicos, 44(2), 329- 351. doi: $10.4067 / \mathrm{s} 0718-07052018000200329$

Stein, E. (2007). La estructura de la persona humana. Madrid: BAC. Stuhr, P., Sutherland, S., \& Ward, P. (2012). Lived-positive emotionality in elementary physical education. Pedagogies: An International Journal, 7(2), 165-181.

Timken, G., McNamee, J. y Sarah, C. (2019). 'It doesn't seem like PE and I love it': Adolescent girl'sviews of a health club physical education approach. European Physical Education Review, 25(1), 1- 16. doi: 10.1177/1356336X17706382

Velázquez, R., \& Hernández, J.L. (2010). La educación física: situación actual y calidad de enseñanza. En R. Velázquez y J. L. Hernández (Eds.), La educación física a estudio. El profesorado, el alumnado y los procesos de enseñanza (pp. 21-36). Barcelona: Graó.

Vendrell, Í. (2015). The emotions in early phenomenology. Studia Phaenomenologica, 15, 349-374. doi: $10.5840 /$ studphaen 20151518

Von Hildebrand, D. (2009). El corazón. Madrid: Palabra.

Vygotsky, L. (1995). Pensamiento y lenguaje. Teoría del desarrollo cultural de las funciones psíquicas. Bogotá: Fausto.

Zaccagnini, J. (2008). La comprensión de la emoción. Una perspectiva psicológica. En M.Jiménez (Ed.), Educación Emocional y Convivencia en el Aula (pp.31-84). Madrid: Ministerio de Educación, Política Social y Deporte.

Zamorano-García, M., Gil-Madrona P., Prieto-Ayuso, A. y ZamoranoGarcía, D. (2018). Generated Emotions By Various Types Of Games In Physical Education. Revista Internacional de Medicinay Ciencias de la Actividad Física y el Deporte, 18(69), 1-26. doi: 10.15366/rimcafd2018.69.001

Zembylas, M. (2019). Intentos por discernir la compleja imbricación entre emoción y pedagogía: contribuciones del giro afectivo. Propuesta Educativa, 28(51), 15-29.

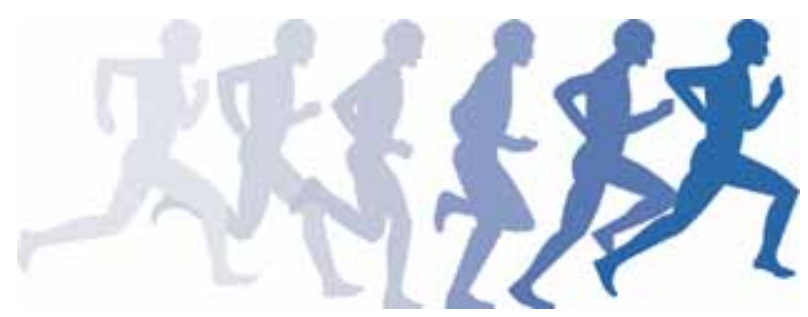

